\title{
Contemporary Cardiovascular Imaging Advancements and Social Media
}

Pedro Covas, MD

Haneen Ismail, MD

Joseph Krepp, MD

Brian G. Choi, MD

Jannet F. Lewis, MD

Richard J. Katz, MD

Andrew D. Choi, MD*

\section{Address}

*Division of Cardiology, The George Washington University School of Medicine, 2150 Pennsylvania Avenue NW, Washington, DC, 20037, USA

Email:adchoi@mfa.gwu.edu

Published online: 15 March 2021

(C) The Author(s), under exclusive licence to Springer Science+Business Media, LLC part of Springer Nature 2021

Pedro Covas and Haneen Ismail contributed equally to this work.

This article is part of the Topical Collection on Imaging

Keywords Cardiac imaging - Social media - Altmetric score - Echocardiography - Nuclear cardiology • Cardio-oncology · Interventional imaging $\cdot$ Computed tomography $\cdot$ Magnetic resonance

\begin{abstract}
Abbreviations AAS Altmetric Attention Score - CCT Cardiovascular computed tomography · CMR Cardiovascular magnetic resonance imaging • ESC European Society of Cardiology • FDA Food and Drug Administration - HIPAA Health Insurance Portability and Accountability Act - ISCHEMIA International Study of Comparative Health Effectiveness with Medical and Invasive Approaches - JACC Journal of the American College of Cardiology - LGE Late gadolinium enhancement - LVEF Left ventricular ejection fraction - MAC Mitral annular calcification - MINOCA Myocardial infarction with nonobstructive coronary arteries - MR Mitral regurgitation - PALS Peak atrial longitudinal strain . PET Positron emission tomography · RVEF Right ventricular ejection fraction . SoMe Social media • TI Transillumination
\end{abstract}

\section{Abstract}

Purpose of review Social media (SoMe) as a means of knowledge dissemination has grown significantly in cardiovascular imaging in recent years. This media platform allows for a free exchange of ideas, the development of new communities, and the ability to disseminate advancements rapidly. While the social media platforms offer limitless potential, their public domain necessitates several important suggestions around best practices. 
Recent findings In cardiovascular imaging, specific hashtags have emerged to encompass the major modalities to include \#EchoFirst, \#YesCCT, \#WhyCMR, and \#CVNuc. Cardiovascular imaging journals have established major presences in the social media space as an avenue to present novel, high-quality, peer-reviewed content to new audiences.

Summary This review paper aims to introduce basic concepts in social media and cardiovascular imaging while highlighting recent topics of high importance, influence, and attention in cardiovascular imaging to include the ISCHEMIA trial, COVID-19, structural imaging, and multimodality advances from throughout 2020.

\section{Introduction}

As cardiovascular imaging advances, knowledge accessibility has evolved to allow impactful clinical developments to disseminate rapidly to the global cardiovascular community. Social media (SoMe) has transformed clinical knowledge sharing of digestible "nuggets of knowledge" that enhance traditional online and inprint media platforms $[1 \bullet \bullet]$. Immediate accessibility and usability of SoMe facilitate novel opportunities for

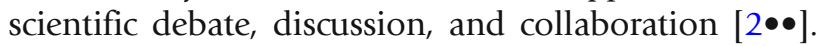
This review paper aims to introduce basic concepts in social media and cardiovascular imaging while highlighting topics of high importance, influence, and attention in cardiovascular imaging from throughout 2020.

\section{Social media engagement}

SoMe networks, including Twitter and Facebook, have grown exponentially within the cardiovascular community over the past several years [3•]. Widespread use of these networks facilitates brief discussions and amplifies attention to contemporary topics [4]. New social media analytic metrics provide a means to measure the initial attention to topics of interest. Specifically, Twitter measures digital "impressions," analogous to speaking to a crowd, to measure uptake, "retweets," and "likes" to measure engagement. The metatags called hashtags (\#) link topics together, facilitate searches, and assess trends [5]. In cardiovascular imaging, specific hashtags have emerged to encompass the major modalities, including \#EchoFirst (e.g., consider echocardiography first line), \#YesCCT (e.g., Yes to appropriate cardiac computed tomography + Society of Cardiovascular CT), \#WhyCMR (e.g., why choose cardiovascular magnetic resonance), and \#CVNuc (nuclear cardiology) [6•]. A number of other new hashtags have trended to include \#CardsRads [7], which presents cardiology and radiology collaborative efforts in advanced cardiovascular imaging, and \#ThePowerofZero, which discusses the role of absent coronary artery calcium de-risking asymptomatic patients at low to intermediate cardiovascular risk [810]. Cardiovascular imaging journals have established major presences in the social media space as an avenue to present novel, high-quality, peer-reviewed content to new audiences [6]. When examining the role of social media in cardiovascular medicine, several recent reports have articulated various angles in the evolution of its use in cardiovascular medicine. Understanding social 
media includes introducing the basics of the platform and best practices [3•].

For clinicians first engaging in this environment, social media offers the opportunity to "navigate the wisdom and madness of the crowd" [11•]. Individual purveyors may find opportunities to amplify their academic work, engage with thought leaders across disciplines, and crowd source perspectives from individual practicing cardiologists, scientists, and cardiovascular imaging advocates. Grassroots advocacy has been provided a new means to create campaigns that collaborate across constituencies. The educator-led "tweetorial," a series of grouped multimedia tweets, may offer opportunities for interactive feedback, polls, and integration of concepts across journals.

Multiple journals have asked whether social media is a "sine qua non," an essential element of knowledge dissemination in both research and case reports $[1 \bullet \bullet, 2 \bullet \bullet]$. Social media has also become an engrained extension of the major national cardiovascular imaging meetings to extend the discussion beyond the meeting walls $[12,13]$. Scholarship has been traditionally assessed in multiple ways. From a journal's perspective, its impact factor, citations, and article downloads and from an author's perspective by metrics such as the h-index, which accounts for the number of overall citations by an author. New social media metrics such as the Altmetric Attention Score (AAS), which is a composite of social media, news articles, blog mentions, and article downloads, and other social media metrics such as the PlumX score, which calculates per social media share as well as hashtag impressions and engagement (shares, likes, and retweets), allow for a nuanced evaluation of article attention (Figs. 1 and 2). The Altmetric Attention Score (AAS) is a metric that measures the total weighted count of the online attention to include social media shares, news articles, and reader shares that a published journal article has received.

To provide reference, AAS of several top cardiovascular journals is included here. Patel et al. compiled the top 10\% of articles in top 4 (by impact factor) major cardiovascular journals (European Heart Journal, Circulation, Journal of the

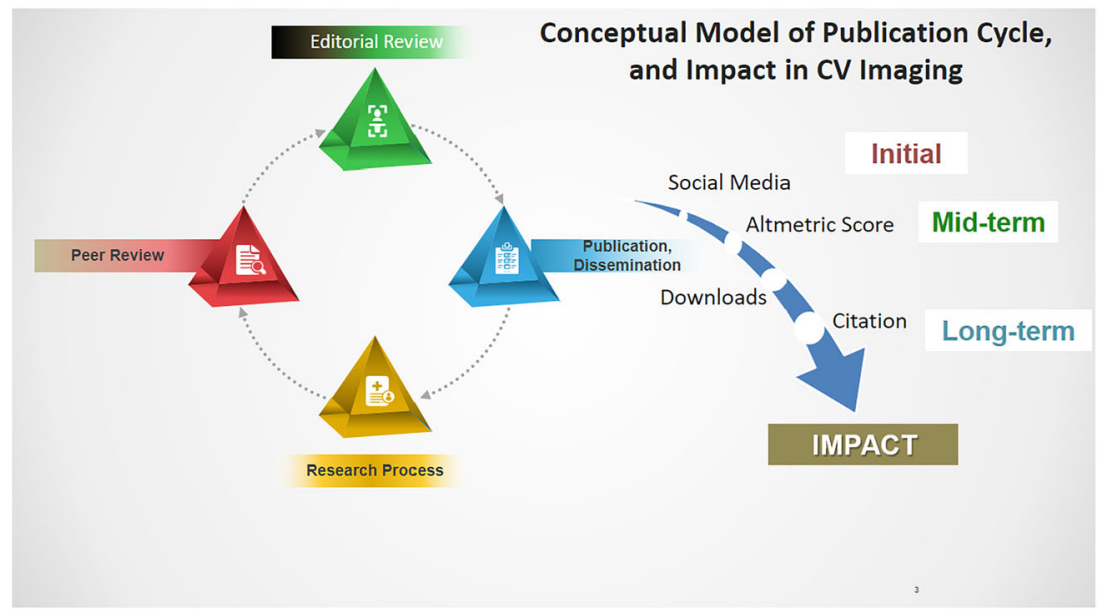

Fig. 1. Conceptual Model of Publication Cycle and Impact in Cardiovascular Imaging. After imaging research, the publication cycle includes peer-review, editorial review, and finally publication. Social media and Altmetrics enable an understanding of the initial (weeks to months) impact of an article, which may have influence on downloads and citations that are measures of impact that become known after months to years. $\mathrm{CV}=$ cardiovascular. Image reproduced with permission from Elsevier. (Choi AD, et al, JACC: Cardiovascular Imaging 2020)(6). 


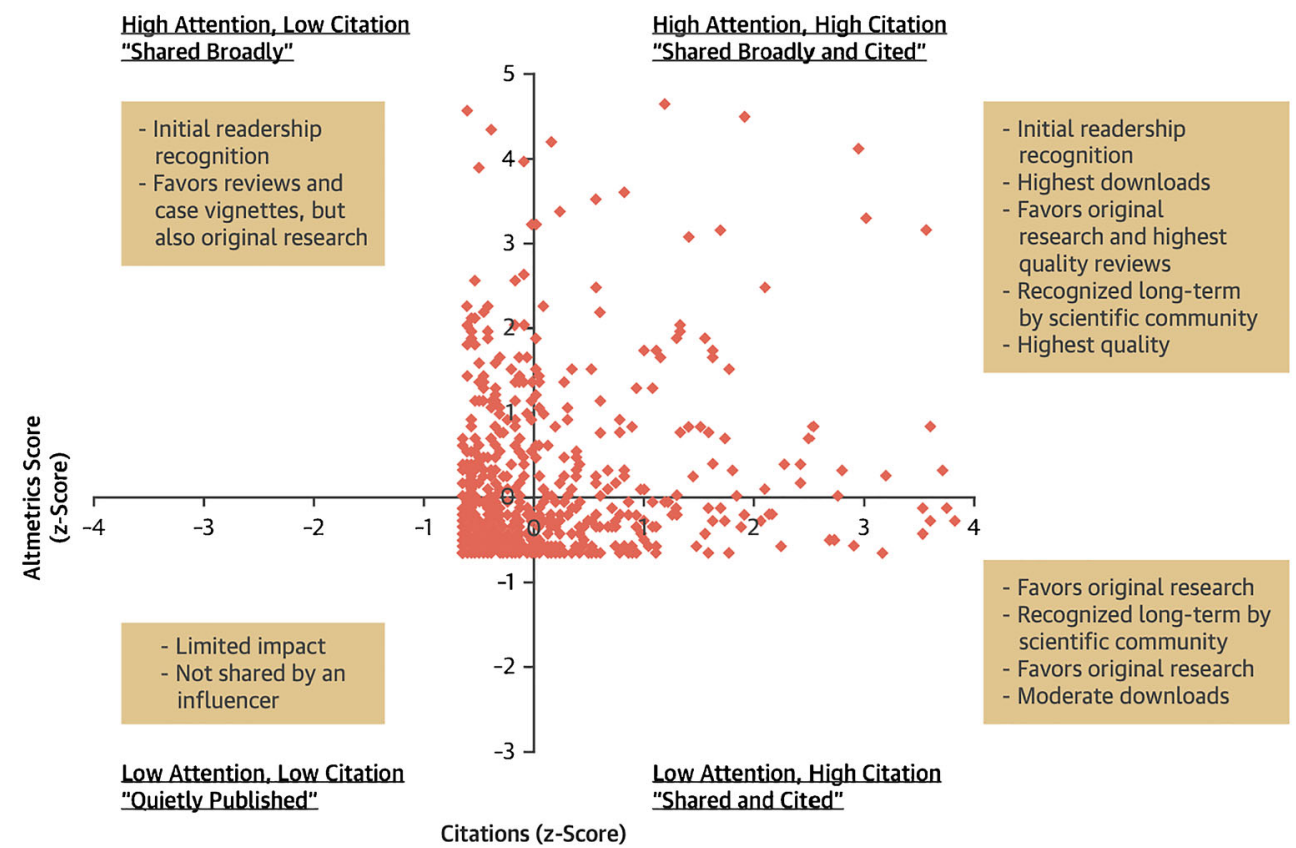

Fig. 2. Conceptual Framework of Altmetrics Versus Citations in CV Imaging. To present a novel model for assessing the impact of new research in cardiovascular imaging, articles published in JACC: Cardiovascular Imaging ( $n=804)$ from 2017 to 2019 were stratified in a $2 \times 2$ contingency table with dots representing an individual article compared with an article's Altmetrics attention score (AAS) and number of total citations. A normalized z-score was calculated for each metric. The articles were divided into: 1 ) high-attention, low citation, termed as "shared broadly"; 2) high-attention, high citation termed as "shared broadly and cited"; 3 ) low attention, low citation termed as "quietly published"; and 4) low attention, high citation termed as "shared and cited with general observations included within the figure. Among the top 100 social media attention articles, AAS median ranged from $36-$ 73. Articles with the highest social media attention and highest citations favored original research from prospective studies and the highest quality invited review articles shared by cardiovascular social media influencers with an outstanding reputation in the field coupled with a large social media followings. Image reproduced with permission from Elsevier. (Choi AD, et al, JACC: Cardiovascular Imaging 2020)(6).

American College of Cardiology, and JAMA: Cardiology), concluding that the median AAS score of these articles was 188 [14]. In JACC: Cardiovascular Imaging, among the top 100 AAS articles from 2017 to 2019, the median AAS was 36-73, whereas all other articles $(n=704)$ had median AAS of 5 [6•]. In JACC: Case Reports, in its first year, the median AAS for clinical cases was $17(n=65), 58.5$

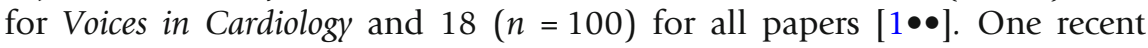
randomized controlled trial from the European Heart Journal reported that papers randomized to a SoMe strategy generated higher AAS and were in turn associated with increased citations [15]. A report from the Journal of the American College of Cardiology suggests that SoMe was associated with increased downloads, suggestive of higher readership [16•]. It is important to note that while higher AAS score reflects higher attention on SoMe, it is unclear whether social media attention reflects a broader adoption of a novel approaches.

The year 2020 was an eventful year for the field of cardiovascular imaging. From this point forward, this article presents a selection of cardiovascular imaging topics generating the highest attention in the past year and their SoMe 
implications, while acknowledging that full coverage of the breadth of social media attention is encouraged through the highlighted references in this brief review article.

\section{COVID-19 and cardiovascular imaging}

Cardiovascular care in 2020 was most notably influenced by the coronavirus pandemic. Coronavirus disease 2019 (COVID-19) impacted society broadly, with important implications for cardiovascular imaging. Novel scholarship focused on how cardiovascular imaging identifies manifestations of COVID19 infection on cardiovascular structure, as well as rapid evolution in clinical practice to encompass new patient and clinician safety concerns [17].

While initial societal guideline documents recommended a cautious approach to cardiovascular imaging of COVID-19 patients, global centers sought to utilize imaging to evaluate the pathology and prognosis in recovered patients. A paper generating extremely high social media attention by Puntmann et al. was published in July 2020 [18•]. In this study of 100 patients with recovered COVID-19 infection, CMR revealed potential cardiac involvement in $78 \%$ of those patients. Moreover, $73 \%$ and $60 \%$ had elevated quantitative T1 and $\mathrm{T} 2$ signal abnormalities, with $32 \%$ of patients demonstrating myocardial fibrosis by late gadolinium enhancement. Compared to healthy controls, those with COVID-19 had a lower LVEF and RVEF. Social media propagated this study to unprecedented levels of attention. With a current Altmetric score of 13,671, and approaching 1 million views, driven by intense interest around college and professional sports decision-making, social media had a significant role in disseminating the findings of myocarditis and CMR. This article is now among the top 30 all-time articles in all research outputs ever recorded by Altmetric and represents the JAMA Cardiology publication with the highest Altmetric score.

Worldwide publicity also brought added scrutiny; this may have contributed to subsequent minor revisions on statistical analysis, methods, and figures to the baseline paper in August 2020 [19]. However, the overall conclusion of the paper remained the same and suggested that CMR identifies myocardial involvement, albeit uncertain prognostic significance. Another small study from China, in 26 post-COVID-19 infection patients $(n=26)$, found that $58 \%$ of patients had myocardial edema by quantitative $\mathrm{T} 1, \mathrm{~T} 2$, and extracellular volume assessment, supporting the findings of Puntmann et al.'s study, and also generated significant social media attention with an Altmetric score of 788 [20].

To address the limitations in these studies, the Ohio State University published a study on CMR findings from athletes with recovered COVID-19 infection [21]. Of the 26 athletes with recovered COVID-19 infection, 12 had CMR evidence of myocarditis or prior myocarditis. This article found a similar prevalence of myocarditis as the previous studies, despite a younger cohort (19.5 years old average). As attention amidst COVID-19 and athletes remained intense, the article generated an Altmetric score of 3571. While this paper informed both the medical and athletic communities of the ongoing cardiac inflammation, it was insufficient to offer uniform consensus on recommendations for return to play in athletes with recovered COVID-19. Subsequent expert consensus statements combined this emerging literature with expert opinion to 
delineate the best way for athletes to safely return to play post-COVID-19 infection through a series of clinical recommendations [22]. This paper highlights the use of multimodality imaging to look for "red flags" that may lead to pathological findings with a current Altmetric score of 827. In December 2020, JACC Cardiovascular Imaging released an expert consensus on screening of competitive athletes recovering from COVID-19 [23]. It highlights the use of cardiac biomarkers, electrocardiograms, and echocardiograms to further risk stratify if CMR and/or other forms of imaging are needed to evaluate the clinical situation. It also emphasizes the limitations of these methods as athletes are a unique population group, and baseline cardiac structure and physiology are often observed from the general population.

In contrast, perhaps highlighting the limitations of attention alone as a quality metric or of ground truth across the population, a small study of competitive athletes $(n=12)$ from Hungary found that none of their postCOVID-19 patients had myocardial abnormalities by CMR T1/T2 quantitative mapping or late gadolinium enhancement imaging. Of note, there has been little social media uptake of research letter with AAS of 0 as of January 2021 [24], emphasizing the well-observed interest in positive studies and less attention to negative findings.

Social media also translated several rapidly produced medical guidelines to meet an urgent need during the COVID-19 pandemic to include protocols for safe and appropriate use of cardiac imaging. The major imaging societies including the American Society of Echocardiography and Society of Cardiovascular Computed Tomography published expert consensus guidance, endorsed by the American College of Cardiology on the optimal use of echocardiograms, both transthoracic and transesophageal, as well as CCT during the pandemic, and with the help of SoMe, the medical community was informed $[25,26]$. Subsequent multimodality guidance followed from the editors of JACC: Cardiovascular Imaging and the European Society of Cardiology and leadership from 16 professional cardiovascular North American Societies [27, 28].

In a time where there was minimal physical interaction throughout the medical community, the COVID-19 pandemic shifted the transfer of medical knowledge to SoMe, as a significant number of physicians became informed through SoMe, demonstrating the influence of SoMe and its versatile use, but with limitations as noted in the example by Vago et al.

\section{ISCHEMIA trial}

Among the most impactful trials performed in cardiovascular imaging is the International Study of Comparative Health Effectiveness with Medical and Invasive Approaches (ISCHEMIA) trial [29]. ISCHEMIA evaluated 5179 patients with moderate to severe ischemia after exclusion of left main disease by coronary computed tomography angiography (CCTA) to assess an invasive versus medical management approach on clinical outcomes. Adherence to medical therapy in both arms was high with $>95 \%$ on statins and aspirin and $77 \%$ with systolic blood pressure $<140 \mathrm{mmHg}$. The study demonstrated that an invasive approach was not associated with a reduction in cardiovascular death, myocardial infarction, hospitalization for unstable angina, heart failure, or resuscitated cardiac arrest over a follow-up of 3.3 years. However, an initial 
invasive strategy was associated with reduced angina and improved quality of life. Several important perspectives of the ISCHEMIA trial have been articulated in detail by Shaw et al. and cardiovascular imaging societies [30•].

In parallel, the hashtag \#ISCHEMIA has garnered exceptionally high attention on social media. From November 2019 to December 2020, \#ISCHEMIA has generated nearly 15,000 tweets from 5500 users generating over 55 million digital impressions (Fig. 3). The broad implications of this trial gave it significant worldwide attention, with the hashtag \#ISCHEMIA trending throughout the end of 2019. The published article currently has 232 citations within a year of publication.

In the midst of this high attention, the social media engagement with the ISCHEMIA trial was perhaps suppressed during the first peak of the COVID-19 pandemic when full study results were published in March 2020 as engagement with the hashtag was highest at the time of the American Heart Association Scientific Sessions with only a modest bump in attention at study publication (Fig. 3). This may also be reflective of the high attention attached to anticipated studies that crest at large international meetings.

While the study did not directly compare imaging strategies, the social media debate has brought several perspectives to light that include the following: (1) challenging the overall purpose of ischemia testing; (2) increased use of CCTA as a front-line test, particularly to exclude high-risk anatomy; and (3) focused role of ischemia imaging in patients with non-specific symptoms for revascularization planning. Blankstein and Shaw note in a broadly shared editorial on social media that perhaps the most important implication of

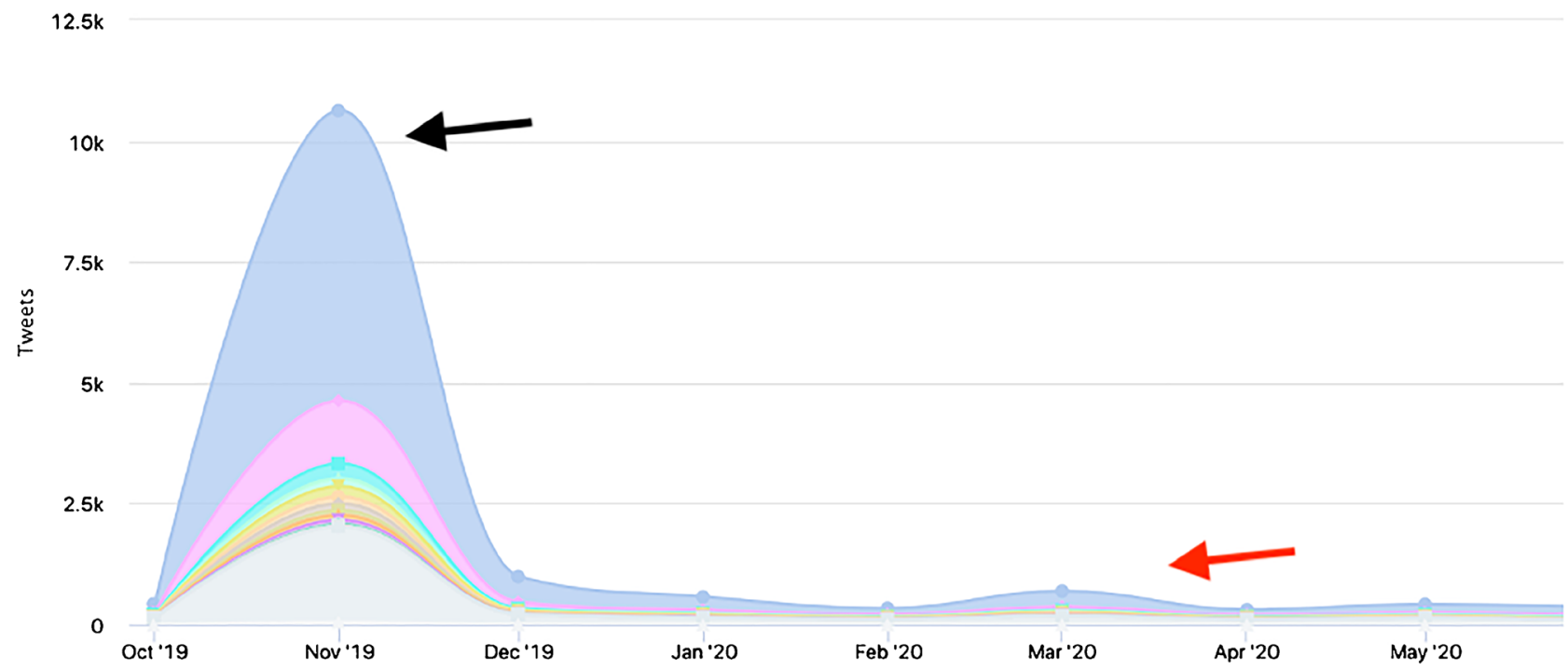

Fig. 3. Social media analytics of \#ISCHEMIA. Social media analytics of the hashtag \#ISCHEMIA (Symplur Signals) demonstrated high social media engagement at the time of the initial presentation of the ISCHEMIA (International Study of Comparative Health Effectiveness with Medical and Invasive Approaches) trial results at the American Heart Association conference in November 2019 (Black Arrow) generating over 10,000 individual tweets and retweets on twitter from predominantly medical professionals. The full publication was released in the New England Journal of Medicine in early April 2020 with modest social media attention as assessed through the \#ISCHEMIA hashtag (Red Arrow). The NEJM publication release coincided with the early stages of the COVID-19 pandemic that may have diminished the response to the landmark cardiovascular trial. 
ISCHEMIA may be enhanced emphasis on an initial strategy of medical therapy in these patients [31].

\section{EVAPORATE trial, MINOCA, and microvascular dysfunction}

Myocardial infarction with nonobstructive coronary artery disease and microvasculature dysfunction have also generated high interest. To highlight a few of topics, the REDUCE-IT trial (Cardiovascular Risk Reduction with Icosapent Ethyl for Hypertriglyceridemia) published in the New England Journal of Medicine demonstrated a significant reduction in the primary composite endpoint of ischemic events, including cardiovascular death for patients receiving icosapent ethyl, and has become an important new therapeutic choice in prevention [32]. A substudy of REDUCE-IT trial entitled EVAPORATE (Effect of Icosapent Ethyl on Progression of Coronary Atherosclerosis in Patients with Elevated Triglycerides on Statin Therapy) was presented at the European Society of Cardiology (ESC) 2020 Congress. This prospective substudy aimed to address the potential linkage between cardiovascular events and non-invasive quantitative plaque analysis [33]. Budoff et al. found that $4 \mathrm{~g}$ /day of icosapent ethyl has significant reduction in total atherosclerotic plaque $(-9 \%)$, high-risk low-attenuation plaque $(-17 \%)$, and total non-calcified plaque $(-19 \%)$ at 18 months of follow-up. These findings and the subsequent debate regarding the magnitude of regression generated high attention with an Altmetric score of 252, reflecting rapid global uptake of these findings. The study serves as a foundation for future trial designs that couple imaging with treatment outcomes.

Myocardial infarction with normal coronary arteries (MINOCA) accounts for $5-6 \%$ of myocardial infarction and yet has important diagnostic and prognostic implications [34]. New advances in 2020 have generated high social media attention.

A recent study by Lintingre et al. (AAS 38) demonstrated that high spatial resolution and free-breathing late gadolinium enhancement (LGE) in 172 consecutive patients with normal initial workup (blood testing, electrocardiogram, echocardiogram, coronary angiogram, traditional CMR) led to a significant increase in confirmed final diagnoses $(26 \%)$ and reduced inconclusive diagnoses (29\%) at the cost of prolonging of the CMR study [35].

Also, at the 2020 ESC Congress, \#whyCMR trended as CMR for evaluation of MINOCA in patients without an obvious underlying cause, particularly to differentiate between ischemic myocardial necrosis and myocardial injury, became a class IB recommendation in new 2020 European Society of Cardiology guidelines (AAS 214) on non-ST elevation acute coronary syndrome [36]. In November 2020, a study by Reynolds with high AAS (138) demonstrated that the use of coronary CT and CMR in women leads to a diagnosis in $84.5 \%$ of participants [37]. The use of both modalities increased diagnoses compared to either one alone. Ischemic and non-ischemic diagnoses were found, while leaving no mechanism for MINOCA in only $15.5 \%$ of patients.

A state-of-the-art review paper by Mathew and colleagues on coronary microvascular dysfunction generated an AAS of 122 and discussed nuances of microvascular blood flow and myocardial perfusion reserve in assessing the microvasculature [38]. They note that cardiac positron emission tomography 
(PET) as well as CMR allows opportunities to directly measure the coronary flow reserve with reduced values associated with worse prognosis.

\section{Structural heart disease}

Structural interventional imaging continues to propagate broad SoMe discussions as structural heart teams seek to incorporate best practices and case experiences from teams around the world. In tricuspid (TR) valve disease, linked through the hashtag \#TreatTR, multiple studies and cases had an early impact through social media. Hahn et al. (AAS 59) discussed the requirements for TR annuloplasty including leaflet devices and transcatheter tricuspid valve replacements [39]. An article by Dreyfus et al. (AAS 33) found that isolated tricuspid valve surgery was associated with high morbidity and mortality, but suggests that early intervention may improve outcome [40]. Quantification and prognostication by CMR-defined stroke volume through phase-contrast methods identified cut-offs of TR volume $\geq 45 \mathrm{~mL}$, or TV regurgitant fraction of $\geq 50 \%$ as prognostically significant also generated significant attention with an Altmetric score of $43[41,42]$. It is expected that this method will impact patient selection for novel tricuspid devices under development such as the TriClip (Abbott, Chicago, IL) or Trialign (Mitralign, Inc., Tewksbury, MA).

An important study in 2020 was one by Guerrero who retrospectively evaluated the use of cardiac computed tomography to risk stratify patients to predict valve embolization prior to potential transcatheter mitral valve replacements in severe mitral annular calcification(MAC) [43]. A scoring system was derived with a summation of 4 parts: calcium thickness, calcium distribution, trigone involvement, and leaflet involvement. Milder MAC scores were $<3$ and severe MAC scores were $\geq 7$. A total of 72 patients with adequate CT scans were retrospectively studied to assess for severity. Interestingly, there was an inverse relationship with increasing MAC scores and degree of migration/embolization. Those with MAC scores $\leq 6$ had a $60 \%$ rate of embolization/migration compared to those with $\geq 7$, which had embolization rates of $9.7 \%$.

Other predictors of low embolization were calcium thickness of $\geq 5 \mathrm{~mm}$, calcium distribution of $>270$ degrees of annular circumference, anterolateral trigone, and anterior leaflet calcification. Mitral regurgitation as the primary pathology was also associated with higher embolization rates. The social media impact was high with an AAS of 91 including being named one of the top 8 cardiac CT studies of the year at the 2020 SCCT Scientific Sessions as highlighted on social media [44]. Of note, many journals such as @JACCJournals will tweet on simultaneous publications from major scientific meetings that drive additional attention to high-quality work.

\section{Echocardiography advances}

Echocardiography continues to have broad discussion on SoMe platforms. Transillumination (TI) is a novel 3D rendering technique that has been developed to provide a virtual light source in the 3D echocardiographic images. A study by Karagodin compared basic 3D images, TI, and TI with transparency of 30 patients representing a variety of structural heart diseases and procedures [45]. Six expert cardiologists and sonographers were asked to grade ability to 
define anatomy, ability to identify pathology, depth perception, and border/ edge delineation. With an array of beautiful figures in the article, TI with transparency was graded as the highest in each of the four categories. Further research aims to provide validation and impact on clinical practice.

Discussion of these visually appealing images has generated significant attention on social media, generating a high PlumX score of 471 , in part driven by interest from individual Twitter users. For this journal (Journal of the American Society of Echocardiography), AAS is not recorded, but PlumX, which accounts only for social media shares, is incorporated. February 2020 brought the most recent focused update on mitral regurgitation (MR) pathways for treatment [46]. Though most of the data is based on the 2014 and 2017 sections, the 2020 updates mainly focus on transcatheter edge-to-edge mitral repair. This is based on information from the COAPT and MITRA-FR studies as well as the FDA approval of the devices for patients with heart failure and secondary MR [47, 48]. The previously used algorithms now include those with secondary MR and new further detailed algorithms for treatment of mitral regurgitation. Other updates include the use of stress echo for determination of symptoms in MR and consideration of rhythm control in those patients with atrial functional MR.

Left atrial strain has become a novel technique in the assessment of diastology. This technique was recently noted to be of prognostic value for developing new atrial fibrillation in heart failure patients [49]. Using dedicated software, peak atrial longitudinal strain (PALS) was assessed through manual tracing of the left atrial endocardial border via speckle tracking. PALS was defined as the first peak positive deflection as representative of left atrial reservoir function. In the STRATS-AHF registry, 397 patients were identified with new atrial fibrillation after left atrial strain imaging on a previous echocardiogram. Lower peak atrial longitudinal strain, defined as less than $18 \%$, was associated with new-onset atrial fibrillation. The researchers found other prognostic factors of developing new atrial fibrillation and developed a scoring system HAS-BEP (Hypertension, Age $>70$, Left Atrial Strain, No beta-blocker, atrial volume index $>40 \mathrm{~mL}$, HFpEF) that may predict future atrial fibrillation. Higher scores tended to have higher association of new atrial fibrillation. This generated an Altmetric score of 32, which is of moderate attention. While this was a high-quality echocardiography study, as reviewed by an editorial board, it may be lower compared to studies discussed within this paper due to its niche topic and differences in attention to research studies, guidelines, and case reports.

\section{Social media best practices and limitations}

While the social media platforms offer limitless potential, their public domain necessitates several important suggestions around best practices (Table 1). Any public account must remain compliant with the Health Insurance Portability and Accountability Act (HIPAA) in the United States or the General Data Protection Rules in the European Union [3•]. Cardiovascular imaging professionals may be held responsible by individual 
Table 1. Cardiovascular Imaging Social Media Suggestions. The table denotes suggestions around best practices in cardiovascular imaging. With regard to case images, it is advised to fully anonymize images, obtain proper permissions that follow national health privacy standards as well as following local individual institutional policies. It is also advised that medical professionals avoid giving specific medical advice on social media

\begin{tabular}{|c|c|c|}
\hline DO's & CAUTION & DON'T'S \\
\hline $\begin{array}{l}\text { Follow your } \\
\text { institutional policy }\end{array}$ & $\begin{array}{l}\text { Controversial topics } \\
\text { (eg politics, religion) }\end{array}$ & $\begin{array}{l}\text { Follow/friend } \\
\text { patients }\end{array}$ \\
\hline$\square$ Identify a focus & $\begin{array}{l}\text { Start slow; don't post } \\
\text { more than } 1 x-2 x / \text { day to } \\
\text { start }\end{array}$ & $\begin{array}{l}\text { Post photos of } \\
\text { patients. }\end{array}$ \\
\hline $\begin{array}{l}\text { Share perspectives \& best } \\
\text { practices }\end{array}$ & $\frac{\text { Post case images }}{\left({ }^{*} \text { obtain permission }\right)}$ & $\Rightarrow \frac{\text { Never post HIPAA }}{\text { identifiable }}$ \\
\hline $\begin{array}{l}\text { Follow the debate on } \\
\text { topics of medical interest }\end{array}$ & $\begin{array}{l}\text { Consider separate } \\
\text { personal vs. } \\
\text { professional profiles }\end{array}$ & $\begin{array}{l}\text { Give specific medical } \\
\text { advice }\end{array}$ \\
\hline $\begin{array}{l}\text { Strive for accuracy, } \\
\text { quality and give credit }\end{array}$ & $\begin{array}{l}\text { Consider adding a } \\
\text { disclaimer }\end{array}$ & $\begin{array}{l}\text { Post something you } \\
\text { wouldn't want to see } \\
\text { in court or in the press } \\
\text { (eg. NY Times) }\end{array}$ \\
\hline$\square$ Be Civil & & \\
\hline
\end{tabular}

institutions in posting content not anonymized and without appropriate permissions as per local institutional policy. Cardiovascular imaging professionals must be cognizant of the legal liability of giving medical advice. Cardiovascular imaging professionals are advised to engage respectfully as offensive or demeaning remarks are not acceptable and may impact the reputation of the clinician, health care center, and team. Cardiovascular imaging professionals are advised to set time limits on use, strive for accuracy, and give appropriate credit to posts. Using educational nuggets, key figures, and visually appealing posts is a means to drive higher engagement with social media. It is also important to ensure that medical professionals engage as a means to provide a credible source of engagement to rebut false narratives (e.g., "fake news") that can quickly propagate within this open environment. At the same time, SoMe does not allow for confidential peer review and information publicized 
may have minimal accountability, which can jeopardize credibility. Paradoxically, post-publication reviews on SoMe may be both informative, but occasionally harsher and off-base when compared to confidential peer-review and editorial board review process. Disclosures of industry relationships or other conflicts of interest are not regulated in SoMe and without clear professional society guidelines it may lead to possible "hidden agendas." One must also be aware that the amount of followers does not always correlate to the level of expertise. Lastly, AAS score may not necessarily correlate with the quality of the paper, as ascertained by an expert editorial board, but rather ascertains the attention it received.

\section{Conclusion}

In conclusion, social media continues to accelerate progress in cardiovascular imaging in a year, 2020, profoundly influenced by the COVID-19 pandemic coupled with multiple advancements across imaging domains. While social media trends may not reflect lasting impact, social media allows the cardiovascular imaging community to understand the early impact of novel work and rapidly disseminate new knowledge. Novel advancements in the field of cardiovascular imaging will continue to disseminate through the limitless potential of social media and contribute to the now globally connected practice of cardiovascular imaging.

\section{Acknowledgements}

The authors wish to acknowledge Drs. Purvi Parwani, Juan Lopez-Mattei, Jeffrey Geske, Ron Blankstein, Leslee Shaw, Y Chandrashekhar, and Todd Villines who have contributed to an understanding of social media metrics in cardiovascular imaging discussed within this paper including development of the figures included with publisher permission in this paper.

\section{Compliance with Ethical Standards}

\section{Conflicts of Interest}

Pedro Covas, Haneen Ismail, Joseph Krepp, Brian G. Choi, Jannet F. Lewis and Richard J. Katz declare that they have no conflict of interest.

Andrew D. Choi reports non-financial support from JACC Journals and non-financial support from Journal of Cardiovascular Computed Tomography, during the conduct of the study as social media editor for these journals.

Human and Animal Rights and Informed Consent

This article does not contain any studies with human or animal subjects performed by any of the authors. 


\section{References and Recommended Reading}

Papers of particular interest, published recently, have been

highlighted as:

- Of importance

• Of major importance

1.• Parwani P, Faza NN, Fudim M, Oliveros E, Brailovsky Y, Choi AD, et al. JACC: case reports: reflections from 1 year on social media. JACC Case Rep. 2020;2(11):1818-23. This study evaluates the impact of social media on a dedicated cardiovascular case reports journal for the first time.

2.• Choi AD, Feuchtner GM, Weir-McCall J, Shaw LJ, Min JK, Villines TC. Accelerating the future of cardiac CT: social media as sine qua non? J Cardiovasc Comput Tomogr. 2020.

This paper evaluates the role and impact of social media on the field of cardiovascular CT for the first time.

3.- Parwani P, Choi AD, Lopez-Mattei J, Raza S, Chen T, Narang A, et al. Understanding social media: opportunities for cardiovascular medicine. J Am Coll Cardiol. 2019;73(9):1089-93.

This paper is a novel white paper that discusses the broad scope of social media through experts in cardiovascular medicine with added expertise in social media through leadership in professional societies and top journals.

4. Walsh MN. Social media and cardiology. J Am Coll Cardiol. 2018;71(9):1044-7.

5. Trueger NS, Bokarius AV, Carroll S, April MD, Thoma B. Impact of a Physician-Led Social Media Sharing Program on a Medical Journal's Web Traffic. J Am Coll Radiol. 2018;15(1 Pt B):184-9.

6. $\quad$ Choi AD, Geske JB, Lopez-Mattei JC, Parwani P, Wang $\mathrm{DD}$, Winchester DE, et al. Cardiovascular imaging through the prism of modern metrics. JACC Cardiovasc Imaging. 2020;13(5):1256-69.

This paper presents a novel model for the intersection of modern metrics, social media and citations in cardiovascular imaging, while summarizing top imaging studies from 20172019.

7. Parwani P, Lopez-Mattei J, Choi AD. Building bridges in cardiology and radiology: why collaboration is the future of cardiovascular imaging. J Am Coll Cardiol. 2018;72(20):2534-8.

8. Mitchell JD, Paisley R, Moon P, Novak E, Villines TC. Coronary artery calcium and long-term risk of death, myocardial infarction, and stroke: the Walter Reed Cohort Study. JACC Cardiovasc Imaging. 2018;11(12):1799-806.

9. Hecht HS, Blaha MJ, Kazerooni EA, Cury RC, Budoff M, Leipsic J, et al. CAC-DRS: coronary artery calcium data and reporting system. An expert consensus document of the Society of Cardiovascular Computed Tomography (SCCT). J Cardiovasc Comput Tomogr. 2018;12(3):185-91.

10. Arnett DK, Blumenthal RS, Albert MA, Buroker AB, Goldberger ZD, Hahn EJ, et al. 2019 ACC/AHA Guideline on the Primary Prevention of Cardiovascular
Disease: a Report of the American College of Cardiology/American Heart Association Task Force on Clinical Practice Guidelines. J Am Coll Cardiol. 2019;74(10):e177-232.

11. Yeh RW. Academic Cardiology and Social Media: Navigating the wisdom and madness of the crowd. Circ Cardiovasc Qual Outcomes. 2018;11(4):e004736.

This paper is one of the first to editorialize the contrast between traditional models of knowledge dissemination versus the social media "crowd" democratization of cardiovascular knowledge discussion.

12. Choi AD, Parwani P, Michos ED, Lee J, Singh V, Fentanes E, et al. The global social media response to the 14th annual Society of Cardiovascular Computed Tomography scientific sessions. J Cardiovasc Comput Tomogr. 2020;14(2):124-30.

13. Aggarwal NR, Bullock-Palmer RP. The voyage: amalgating a social media platform through the annual scientific meeting. J Nucl Cardiol. 2020;27(1):18-24.

14. Patel RB, Vaduganathan M, Bhatt DL, Bonow RO. Characterizing high-performing articles by Altmetric score in major cardiovascular journals. JAMA Cardiol. 2018;3(12):1249-51.

15. Ladeiras-Lopes R, Clarke S, Vidal-Perez R, Alexander M, Luscher TF. Twitter promotion predicts citation rates of cardiovascular articles: a preliminary analysis from the ESC Journals Randomized Study. Eur Heart J. 2020;41(34):3222-5.

16. Parwani P, Martin GP, Mohamed MO, Hajeer A, Nwaokoro M, Narang A, et al. Relationship of Altmetric attention score to overall citations and downloads for papers published in JACC. J Am Coll Cardiol. 2020;76(6):757-9.

This paper directly assesses altmetrics attention score to citations in JACC.

17. Guan WJ, Ni ZY, Hu Y, Liang WH, Ou CQ, He JX, et al. Clinical characteristics of coronavirus disease 2019 in China. N Engl J Med. 2020;382(18):1708-20.

18. Puntmann VO, Carerj ML, Wieters I, Fahim M, Arendt C, Hoffmann J, et al. Outcomes of cardiovascular magnetic resonance imaging in patients recently recovered from coronavirus disease 2019 (COVID-19). JAMA Cardiol. 2020;5(11):1265-73.

This paper received extremely high attention for findings of myocardial involvement in recovered COVID-19 patients by cardiac MRI.

19. Nagel E, Puntmann VO. Errors in statistical numbers and data in study of cardiovascular magnetic resonance imaging in patients recently recovered from COVID19. JAMA Cardiol. 2020;5(11):1307-8. 
20. Huang L, Zhao P, Tang D, Zhu T, Han R, Zhan C, et al. Cardiac involvement in patients recovered from COVID-2019 identified using magnetic resonance imaging. JACC Cardiovasc Imaging. 2020;13(11):23309.

21. Rajpal S, Tong MS, Borchers J, Zareba KM, Obarski TP, Simonetti OP, et al. Cardiovascular magnetic resonance findings in competitive athletes recovering from COVID-19 infection. JAMA Cardiol. 2020.

22. Kim JH, Levine BD, Phelan D, Emery MS, Martinez MW, Chung EH, et al. Coronavirus disease 2019 and the athletic heart: emerging perspectives on pathology, risks, and return to play. JAMA Cardiol. 2020.

23. Phelan D, Kim JH, Elliott MD, Wasfy MM, Cremer P, Johri AM, et al. Screening of potential cardiac involvement in competitive athletes recovering from COVID19: an expert consensus statement. JACC Cardiovasc Imaging. 2020;13(12):2635-52.

24. Vago H, Szabo L, Dohy Z, Merkely B. Cardiac magnetic resonance findings in patients recovered from COVID19: initial experiences in elite athletes. JACC

Cardiovasc Imaging 2020.

25. Choi AD, Abbara S, Branch KR, Feuchtner GM, Ghoshhajra B, Nieman K, et al. Society of Cardiovascular Computed Tomography guidance for use of cardiac computed tomography amidst the COVID-19 pandemic endorsed by the American College of Cardiology. J Cardiovasc Comput Tomogr. 2020;14(2):101-4.

26. Kirkpatrick JN, Mitchell C, Taub C, Kort S, Hung J, Swaminathan M. ASE statement on protection of patients and echocardiography service providers during the 2019 novel coronavirus outbreak: endorsed by the American College of Cardiology. J Am Soc Echocardiogr. 2020;33(6):648-53.

27. Zoghbi WA, DiCarli MF, Blankstein R, Choi AD, Dilsizian V, Flachskampf FA, et al. Multimodality cardiovascular imaging in the midst of the COVID-19 pandemic: ramping up safely to a new normal. JACC Cardiovasc Imaging. 2020;13(7):1615-26.

This is an important JACC: CV Imaging / ACC Imaging guidance document for imaging best practices amidst COVID-19.

28. Wood DA, Mahmud E, Thourani VH, Sathananthan J, Virani A, Poppas A, et al. Safe reintroduction of cardiovascular services during the COVID-19 pandemic: from the North American Society Leadership. J Am Coll Cardiol. 2020;75(25):3177-83.

29. Maron DJ, Hochman JS, Reynolds HR, Bangalore S, O'Brien SM, Boden WE, et al. Initial invasive or conservative strategy for stable coronary disease. $\mathrm{N}$ Engl J Med. 2020;382(15):1395-407.

30. Shaw L, Kwong RY, Nagel E, Salerno M, Jaffer F, Blankstein $\mathrm{R}$, et al. Cardiac imaging in the postISCHEMIA trial era: a multisociety viewpoint. JACC Cardiovasc Imaging. 2020;13(8):1815-33.

This paper presents a broad multisociety perspective on the implications of the ISCHEMIA trial for each imaging modality.
31. Blankstein R, Shaw LJ. Ischemia trial: implications for coronary CT angiography. J Cardiovasc Comput Tomogr. 2020;14(1):1-2.

32. Bhatt DL, Steg PG, Miller M, Brinton EA, Jacobson TA, Ketchum SB, et al. Cardiovascular risk reduction with Icosapent ethyl for hypertriglyceridemia. $\mathrm{N}$ Engl J Med. 2019;380(1):11-22.

33. Budoff MJ, Bhatt DL, Kinninger A, Lakshmanan S, Muhlestein JB, Le VT, et al. Effect of icosapent ethyl on progression of coronary atherosclerosis in patients with elevated triglycerides on statin therapy: final results of the EVAPORATE trial. Eur Heart J. 2020;41:3925-32.

34. Tamis-Holland JE, Jneid H, Reynolds HR, Agewall S, Brilakis ES, Brown TM, et al. Contemporary diagnosis and management of patients with myocardial infarction in the absence of obstructive coronary artery disease: a scientific statement from the American Heart Association. Circulation. 2019;139(18):e891-908.

35. Lintingre PF, Nivet H, Clement-Guinaudeau S, Camaioni C, Sridi S, Corneloup O, et al. Highresolution late gadolinium enhancement magnetic resonance for the diagnosis of myocardial infarction with nonobstructed coronary arteries. JACC Cardiovasc Imaging. 2020;13(5):1135-48.

36. Collet JP, Thiele H, Barbato E, Barthelemy O, Bauersachs J, Bhatt DL, et al. ESC guidelines for the management of acute coronary syndromes in patients presenting without persistent ST-segment elevation. Eur Heart J. 2020;2020.

37. Reynolds HR, Maehara A, Kwong RY, Sedlak T, Saw J, Smilowitz NR, et al. Coronary optical coherence tomography and cardiac magnetic resonance imaging to determine underlying causes of MINOCA in women. Circulation. 2020.

38. Mathew RC, Bourque JM, Salerno M, Kramer CM. Cardiovascular imaging techniques to assess microvascular dysfunction. JACC Cardiovasc Imaging. 2020;13(7):1577-90.

39. Hahn RT, Nabauer M, Zuber M, Nazif TM, Hausleiter J, Taramasso $\mathrm{M}$, et al. Intraprocedural imaging of transcatheter tricuspid valve interventions. JACC Cardiovasc Imaging. 2019;12(3):532-53.

40. Dreyfus J, Flagiello M, Bazire B, Eggenspieler F, Viau F, Riant E, et al. Isolated tricuspid valve surgery: impact of aetiology and clinical presentation on outcomes. Eur Heart J. 2020;41(45):4304-17.

41. Zhan Y, Senapati A, Vejpongsa P, Xu J, Shah DJ, Nagueh SF. Comparison of echocardiographic assessment of tricuspid regurgitation against cardiovascular magnetic resonance. JACC Cardiovasc Imaging. 2020;13(7):1461-71.

42. Zhan Y, Debs D, Khan MA, Nguyen DT, Graviss EA, Khalaf S, et al. Natural history of functional tricuspid regurgitation quantified by cardiovascular magnetic resonance. J Am Coll Cardiol. 2020;76(11):1291-301.

43. Guerrero M, Wang DD, Pursnani A, Eleid M, Khalique $\mathrm{O}$, Urena $\mathrm{M}$, et al. A cardiac computed tomographybased score to categorize mitral annular calcification 
severity and predict valve embolization. JACC

Cardiovasc Imaging. 2020;13(9):1945-57.

44. Weir-McCall JR, Branch K, Ferencik M, Blankstein R, Choi AD, Ghoshhajra BB, et al. Highlights of the 15th annual scientific meeting of the Society of Cardiovascular Computed Tomography. J Cardiovasc Comput Tomogr. 2020

45. Karagodin I, Addetia K, Singh A, Dow A, Rivera L, DeCara JM, et al. Improved delineation of cardiac pathology using a novel three-dimensional echocardiographic tissue transparency tool. J Am Soc Echocardiogr. 2020;33(11):1316-23.

46. Bonow RO, O'Gara PT, Adams DH, Badhwar V, Bavaria JE, Elmariah S, et al. 2020 Focused Update of the 2017 ACC expert consensus decision pathway on the management of mitral regurgitation: a report of the American College of Cardiology Solution Set Oversight Committee. J Am Coll Cardiol. 2020;75(17):2236-70.

47. Obadia JF, Messika-Zeitoun D, Leurent G, Iung B, Bonnet G, Piriou N, et al. Percutaneous repair or medical treatment for secondary mitral regurgitation. N Engl J Med. 2018;379(24):2297-306.

48. Stone GW, Lindenfeld J, Abraham WT, Kar S, Lim DS, Mishell JM, et al. Transcatheter mitral-valve repair in patients with heart failure. N Engl J Med. 2018;379(24):2307-18.

49. Park JJ, Park JH, Hwang IC, Park JB, Cho GY, Marwick $\mathrm{TH}$. Left atrial strain as a predictor of new-onset atrial fibrillation in patients with heart failure. JACC Cardiovasc Imaging. 2020;13(10):2071-81.

\section{Publisher's Note}

Springer Nature remains neutral with regard to jurisdictional claims in published maps and institutional affiliations. 